\title{
Development of wide-range constitutive equations for calculations of high-rate deformation of metals
}

\author{
V.A. Raevsky ${ }^{1, a}$, O.N. Aprelkov ${ }^{1}$, O.N. Ignatova ${ }^{1}$, V.I. Igonin ${ }^{1}$, A.I. Lebedev ${ }^{1}$, S.S. Nadezhin ${ }^{1}$, \\ M.A. Zocher ${ }^{2}$, D. Preston ${ }^{2}$, and A. Coul ${ }^{2}$ \\ 1 FSUE "RFNC - VNIIEF", Sarov, Russia \\ 2 Los Alamos National Laboratory, Los Alamos, USA
}

\begin{abstract}
For development of models of strength and compressibility of metals in wide range of pressures (up to several megabar) and strain rates $\dot{\varepsilon} \sim 1 \div 10^{8} \mathrm{~s}^{-1}$, the method of dynamic tests is used. Since direct measurement of strength is impossible under complicated intensive high-rate loading, a formal model is created at first, and then it is updated basing on comparison with many experiments, which are sensitive to shear strength. Elastic-plastic, viscous-elastic-plastic and relaxation integral models became nowadays most commonly used. The basic unsolved problems in simulation of high-rate deformation of metals are mentioned in the paper.
\end{abstract}

\section{Introduction}

Since the sixties-seventies of the previous century, scientists from various countries have been involved in development of models of material behavior at high strain rate [1-8]. However, though there is such a history and achievements of present-day numerical methods, development of equations, which determine relations between stress tensor and strain tensor, is currently still an unsolved urgent problem. Reason is, first of all, complexity of deformation process. It includes competition of various strain mechanisms, structural changes during deformation, violation of flow stability at various scale levels, etc. [9-11]. On the one hand, models for calculations of high-velocity dynamic processes should be rather simple and physically clear; on the other hand, they should reflect the main processes, which occur in materials, and to "work" in wide range of flow parameters. In particular, constitutive equations should reflect the dependence of stress tensor on flow parameters at certain time and on strain history. In our understanding, strain history means the dependences of temperature, pressure, strain tensor and stress tensor on time. Strain history determines structural changes, namely, growth of dislocation density, changes of sizes and orientation of grains, relative sliding of grains, formation of twin structures and bands of localized shift, annealing when reaching critical temperatures [9, 11-13].

Elastic-plastic, viscous-elastic-plastic and relaxation integral models became nowadays most commonly used.

\section{Relaxation model of strength}

Simplified relaxation model is based on usual elastic-plastic approximation

$$
\frac{2}{3} Y_{d}^{2} \geq S_{i j} \cdot S_{i j}
$$

\footnotetext{
a e-mail: root@gdd.vniief.ru
}

This is an Open Access article distributed under the terms of the Creative Commons Attribution-Noncommercial License 3.0, which permits unrestricted use, distribution, and reproduction in any noncommercial medium, provided the original work is properly cited. 


\section{EPJ Web of Conferences}

and dynamic yield strength can be determined by the differential equation, which takes account for the process of relaxation of elastic stress

$$
\dot{Y}_{d}=3 G \dot{\varepsilon}_{i}-\frac{Y_{d}-Y_{S}}{\tau}
$$

where $S_{i j}$ is the deviatoric component of stress; $\dot{\varepsilon}_{i}$ is the strain rate; $G$ is the shear modulus; $\tau$ is the time of relaxation of elastic stress; $Y_{d}$ is the effective yield strength at certain time; $Y_{S}$ is the stationary yield strength, which occurs in this state $(P, T)$ of substance at $\dot{\varepsilon}_{i}=0$. Points above $Y_{d}$ and $\varepsilon_{i}$ mean time derivative. Equation (1) is obtained from the known relation for the deviatoric component of stress

$$
\dot{S}_{i j}=2 \cdot G \cdot\left(\dot{e}_{i j}-\dot{\varepsilon}_{i j}^{P}\right),
$$

with the supposition that the deviatoric component of strain rate $\dot{e}_{i j}$ is collinear to tensor of plastic strain rate $\dot{\varepsilon}_{i j}^{P}$.

At sufficiently slow change of the yield strength $\left(\dot{Y}_{d} \approx 0\right)$, Eq. (1) transits to the elastic-viscousplastic model

$$
Y_{d}=Y_{S}+3 \cdot G \cdot \tau \cdot \dot{\varepsilon}_{i}
$$

Contrary to the relaxation model of Steinberg [7], Eq. (1) is much simpler, since it contains only one equation instead of three. Besides, to solve the problem of deformation, it is enough to use a usual elastic-plastic model with $Y_{d}$ variable in time. For determination of the stationary yield strength, the equation of the type of Zerilli F.J., Armstrong R.W [3] is used:

$$
Y_{S}=Y_{0} \cdot\left[1+a_{0} \cdot\left(1-\bar{T}^{k}\right) \cdot\left(1-\exp \left(-\frac{\varepsilon_{i}^{p}}{\varepsilon_{1}}\right)\right)\right] \cdot \frac{G}{G_{0}}
$$

or

$$
Y_{S}=Y_{0} \cdot(1+f(t)) \cdot \frac{G}{G_{0}},
$$

Available experimental data point to significant structural changes of metals depending on loading type, and, as a result, change of mechanical properties [9-14]. Thus, intensive deformation of coarsegrain copper causes significant change of grain sizes at low pressure and low strain rate. According to the Hall-Petch law, it results in increase of the conventional yield strength. Increase of strain rate to some critical values $\left(\sim 10^{7} \mathrm{~s}^{-1}\right)$ causes fast growth of dislocation density and formation of twins, which can be aggregated as packages consisting of tens of parallel micro twins $[11,16]$. In this case, the stationary yield strength grows much quicker than during slow (static) deformation, approaching its limiting value. Intensive shock waves cause temperature increase that can anneal metal. Dislocations will be annihilated, and strength will be reduced, respectively. Models are presently under development, which take account for dependence of hardening on strain rate, as well as softening due to heating [15]. One of variants of these models has the following form.

$$
\dot{f}(t)=\frac{\varphi\left(\dot{\varepsilon}_{i}\right) \cdot \dot{\varepsilon}_{i}}{\varepsilon_{1}} \cdot\left(f_{a}(0)-f\right)-\frac{f-f_{a}(\bar{T})}{\tau_{a}} \cdot \Theta\left(\frac{f}{f_{a}(\bar{T})}-1\right) .
$$

The first part in Eq. (3), on the right, takes integrally account for hardening, which is associated with formation of defects (dislocations, new boundaries of grains, and formation of twin structures at high strain rate $\left(\dot{\varepsilon}_{i}>10^{7} \mathrm{~s}^{-1}\right)$. Function $\varphi\left(\dot{\varepsilon}_{i}\right)$ takes account for nonlinear dependence of hardening on strain rate. The second part in equation (3), on the right, takes account for temperature annealing or annihilation of dislocations for time of temperature relaxation $\tau_{a} ; \Theta$ - the Heaviside function $(\Theta=0$ at $f<f_{a}$ and $\Theta=1$ at $f \geq f_{a}$ ). Experiments with study of change of material properties after effect of high-rate deformation and shock waves are used for determination of functions $\varphi\left(\dot{\varepsilon}_{i}\right), \tau_{a}$ [9-12]. 
New Models and Hydrocodes for Shock Wave Processes in Condensed Matter

Table 1. Constant coefficients of equations (5)-(6).

\begin{tabular}{|c|c|c|c|c|c|c|c|c|c|c|}
\hline Metal & $\boldsymbol{\rho}_{\mathbf{0} \boldsymbol{k}}, \mathbf{g} / \mathbf{c m}^{3}$ & $\boldsymbol{\rho}_{\mathbf{0}}, \mathbf{g} / \mathbf{c m}^{3}$ & $\boldsymbol{\rho}_{\boldsymbol{m} \mathbf{0}}, \mathbf{g} / \mathbf{c m}^{3}$ & $\boldsymbol{C}_{\mathbf{0} \boldsymbol{k}}, \mathbf{m} / \mathbf{s}$ & $\boldsymbol{n}$ & $\boldsymbol{\Gamma}_{\boldsymbol{\infty}}$ & $\boldsymbol{\Gamma}_{\mathbf{0}}$ & $\boldsymbol{M}$ & $\boldsymbol{c}_{\boldsymbol{V}}, \mathbf{J} / \mathbf{g K}$ & $\boldsymbol{T}_{\boldsymbol{m} \mathbf{0}}, \mathbf{K}$ \\
\hline $\mathrm{Al} \mathrm{AMg-6}$ & 2.69 & 2.64 & 2.524 & 5.55 & 3.2 & 0.67 & 2.14 & 1.2 & 0.9 & 933 \\
\hline $\mathrm{Cu}$ & 9.054 & 8.93 & 8.384 & 3.95 & 4.3 & 0.7 & 1.96 & 1.2 & 0.391 & 1356 \\
\hline $\mathrm{Ta}$ & 16.76 & 16.659 & 15.478 & 3.3 & 3.4 & 0.67 & 1.7 & 1.0 & 0.14 & 3290 \\
\hline
\end{tabular}

Table 2. Constant coefficients of equations (1)-(4).

\begin{tabular}{|c|c|c|c|c|c|c|c|c|c|c|}
\hline Metal & $\boldsymbol{Y}_{\mathbf{0}}, \boldsymbol{G P a}$ & $\boldsymbol{a}_{\mathbf{0}} \boldsymbol{f}_{a}(\mathbf{0})$ & $\boldsymbol{k}$ & $\varepsilon_{1}$ & $\boldsymbol{G}_{0}, \boldsymbol{G P a}$ & $\boldsymbol{\tau}_{\mathbf{0}}, \mu \mathbf{S}$ & $\dot{\boldsymbol{\varepsilon}}_{\boldsymbol{i 0}}, 1 / \mathbf{s}$ & $\boldsymbol{\varphi}_{\max }$ & $\dot{\boldsymbol{\varepsilon}}_{\mathbf{0 S}}, \mathbf{1} / \mathbf{s}$ & $\boldsymbol{n}$ \\
\hline $\mathrm{Al} \mathrm{AMg-6}$ & 0.205 & 1.4 & 5 & 0.1 & 28 & 2.28 & 1.0 & 0 & - & 0 \\
\hline $\mathrm{Cu}$ & 0.055 & 9.66 & 1.0 & 0.66 & 40 & 25 & 0.25 & 50 & $10^{6}$ & 2 \\
\hline $\mathrm{Ta}$ & 0.44 & 1.4 & 1.0 & 0.5 & 70 & 5.0 & 0.5 & 0 & - & 0 \\
\hline
\end{tabular}

To determine relaxation time $\tau$, experiments of Barker and Johnson are used [16], as well as experiments by the Hopkinson method [13], with compression of cylindrical shells [17], as well as research of collapse of cylindrical channels having small cross-sections [14]. We are using the dependence for relaxation time $\tau$, which takes account for compression, temperature, and strain rate in the following form:

$$
\tau=\frac{\tau_{0} \cdot(1-\bar{T})^{2}}{\frac{G}{G_{0}} \cdot\left(1+\frac{\dot{\varepsilon}_{i}}{\dot{\varepsilon}_{i 0}} \cdot \varphi\left(\dot{\varepsilon}_{i}\right)\right)^{0.5}}, \quad \varphi\left(\dot{\varepsilon}_{i}\right)=\frac{1+\varphi_{\max } \cdot\left(\frac{\dot{\varepsilon}_{i}}{\dot{\varepsilon}_{0 S}}\right)^{n}}{1+\left(\frac{\dot{\varepsilon}_{i}}{\dot{\varepsilon}_{0 S}}\right)^{n}} .
$$

Equation (4) has the following limiting values: at $\dot{\varepsilon}_{i} \rightarrow 0, G=G_{0} \tau=\tau_{0}$, at $\bar{T} \rightarrow 1$ or $\dot{\varepsilon}_{i} \rightarrow \infty \tau \rightarrow 0$.

In the area of comparatively low pressures $(<100 \mathrm{GPa})$, the equation of state can be presented in the simplified Mie-Grueneisen form:

$$
\begin{gathered}
P=\frac{\rho_{0 K} C_{0 K}^{2}}{n}\left(\delta^{n}-1\right)+\Gamma_{P} \cdot \rho \cdot E_{T} . \\
\Gamma_{p}=\Gamma_{\infty}+\frac{\Gamma_{0}-\Gamma_{\infty}}{\delta^{M}},
\end{gathered}
$$

In relations (5), (6) $\rho_{0 K}, C_{0 K}$ are the density and the volume sound velocity at $T=0 K ; \frac{\rho_{0 K} C_{0 K}^{2}}{n}\left(\delta^{n}-1\right)=$ $P_{e}$ is the elastic component of pressure; $\Gamma_{P}$ is the Grueneisen coefficient; $\rho$ is the substance density; $\delta=\frac{\rho}{\rho_{0 k}}$ is relative compression; $E_{T}$ are internal energies, $\Gamma_{0}, \Gamma_{\infty}$ are values of the Grueneisen coefficient $\left(\Gamma_{P}\right)$ at $\rho=\rho_{0 K}$ and $\delta \rightarrow \infty$, respectively.

Tables 1, 2 present parameters of relaxation strength models and equations of state for copper, tantalum and aluminum alloy AMg-6 (aluminum 6061).

Relaxation models give satisfactory description of the following peculiarities of dynamic deformation: strain hardening, nonlinear dependence of hardening velocity on strain rate, thermal softening, viscosity-increase of current stress as strain rate grows, compression hardening.

\section{Experiments for justification of strength models and some results of simulation}

As a rule, it is impossible to determine yield strength basing directly on experiments. The value, which is sensitive to shear strength, is measured in the experimental techniques, which are known for the 


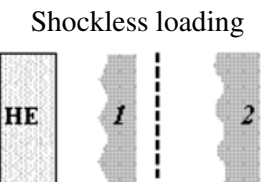

Shock-wave loading

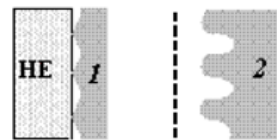

1 - investigated liner, 2 - liner during loading

a)
Protonographic images

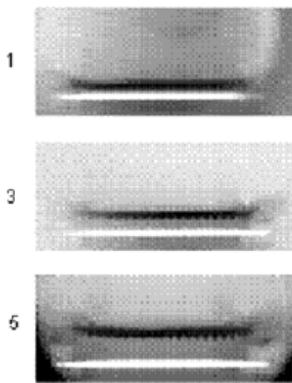

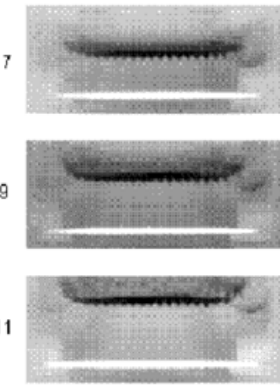

b)

Fig. 1. Method for radiographic recording of perturbation growth: a - loading method; $b$, - examples of photos.

authors. To determine the yield strength, the numerical-experimental method is used. Its essence is the following. Having specified physical and mathematical forms of the constitutive equation, selection or correction of numerical parameters of mathematical model is performed by comparison of experimental and numerical results, when adequate description is provided by calculation of primary experimental data. Wider is class of experiments used for testing (from static experiments to shockwave experiments), more precise is the selected form of model and parameters. Finally, using the numerical-experimental method, it is possible to determine metal yield strength in the investigated strain area. Precision of shear strength value, which is determined by this method, depends on precision of parameters measured in experiments.

Parametric identification of Eqs. (1)-(4) was performed basing on various experiments, namely, compression diagrams, Taylor method, compression of cylindrical channels, method of perturbation growth recording, analysis of release wave structure, measurement of elastic precursor, measurement of shock wave front width, as well as basing on metallographic and mechanical researches of samples after shock-wave loading [9-21]. These methods are briefly listed below.

For the shock-wave regime of deformation, it is possible to observe faster hardening than that in the quasi-static regime. At pressure $P \sim 30-40 \mathrm{GPa}$, strength reaches the maximum, and it is reduced as pressure continues growing. It is caused by annealing behind shock wave front. And annealing value depends not only on temperature behind the front, but on duration of shock-wave loading $\tau_{d t}$.

One of the methods for checking the strength models in the area of high strain rate is based on comparison of calculations and experiments for strain rate measurement in front of shock waves [16]. At certain selection of dependence of relaxation time on strain rate, for example, in form (4), it is possible to describe experimental results.

The other, comparatively new method for testing the high-velocity component of dynamic strength includes numerical simulation of convergence of cylindrical apertures having small diameters under effect of planar shock waves [14]. Radiographic and proton radiography methods of perturbations allow testing strength models in wide ranges of pressures $\left(P \sim 10-10^{3} \mathrm{GPa}\right)$ and strain rates $\left(\dot{\varepsilon}_{i} \sim 10^{4}-\right.$ $\left.10^{7} \mathrm{~s}^{-1}\right)$ [20,21]. Figure 1 presents schemes for loading the investigated samples and X-ray photos of the samples in motion, which were obtained by various techniques.

Example of numerical description of perturbation growth is shown in Fig. 2.

\section{Heterogeneous model of strength}

However, there are some effects, which are not taken into account by the above-described models. Among them, the Bauschinger's effect and the effect of temporal strength loss in some metals, which is caused by growth of heterogeneous structures at high-rate deformation and, in particular, after effect of shock waves. These structures appear during strain with rate higher $\approx 10^{7} \mathrm{~s}^{-1}$. Formation of these 


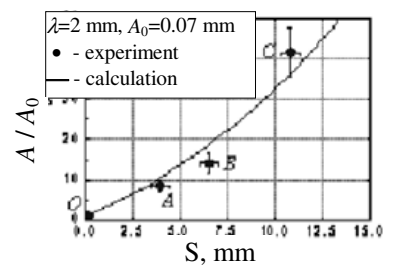

Perturbation growth

\section{mn}

Experiment / calculation (C)

\section{mrrm ${ }^{c}$}
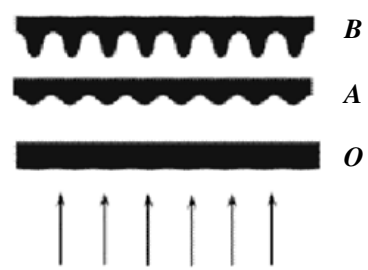

Loading by detonation products

Fig. 2. Numerical simulation of perturbation growth in copper sample.

Shockless loading $\dot{\varepsilon} \sim 10^{6} \mathrm{~s}^{-1}$

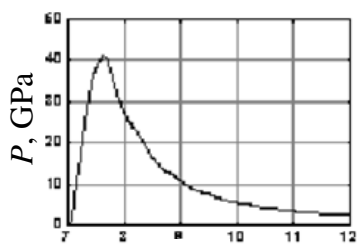

$t, \mu \mathrm{s}$

Shock-wave loading $\dot{\varepsilon} \sim 10^{8} \mathrm{~s}^{-1}$

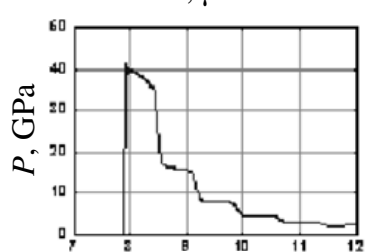

$t, \mu \mathrm{s}$

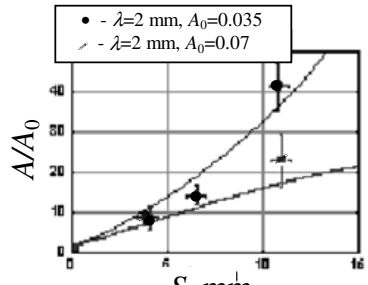

$S, \mathrm{~mm}$

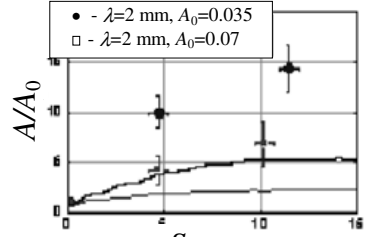

$S, \mathrm{~mm}$

Pressure versus time

b) $\mathrm{Cu}$.

Perturbation growth

Fig. 3. Calculations of perturbation growth with use of relaxation model.

structures can be accompanied with temporal reduction of stress intensity. Strength drop during strain rate increase is not described by usual models of strength, including by the relaxation model. Figure 3 presents results of calculations of perturbation growth on copper liner surface under shock-wave and shockless loadings.

As it is possible to see in the graphs, calculations provide good description of perturbation growth in copper and aluminum liners under shockless loading, and they significantly underestimate perturbation growth under shock-wave loading. Strengths of shock-loaded and recovered samples are even higher than those loaded in the shockless regime. Therefore, strength drop has a temporal character. Numerical analysis of experiments showed that the softening time is $t \sim 0.3-0.4 \mu$ s for aluminum and copper.

D. Gready [8] made a supposition that the phenomenon of temporal loss of strength occurs as a result of formation of layers with increased temperature behind shock wave front. As many experiments revealed, bands of localized shift of the twin nature appear in samples after effect of shock waves of sufficient intensity. To some extent, it confirms the D. Gready's hypothesis.

Basing on this hypothesis, we developed a two-temperature relaxation model of strength, which takes account for heterogeneous character of deformation and temporal softening [22]. The model is based on the supposition about deformed medium, which consists of periodic layers of substance with 
EPJ Web of Conferences

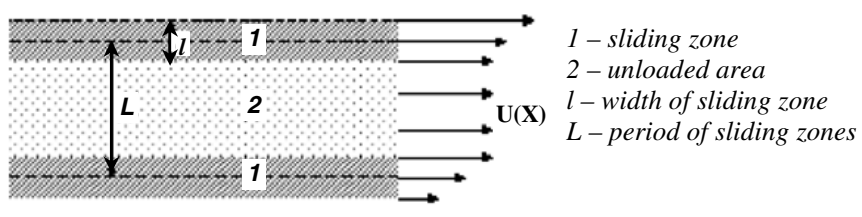

Fig. 4. Model of heterogeneous flow.

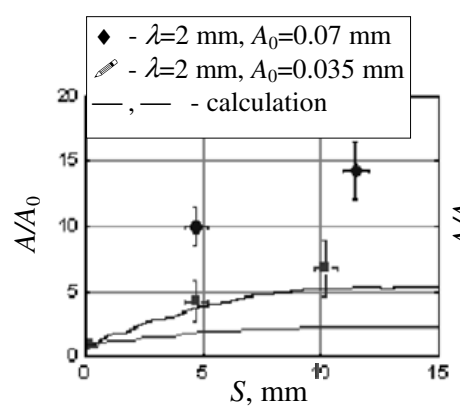

Homogeneous model

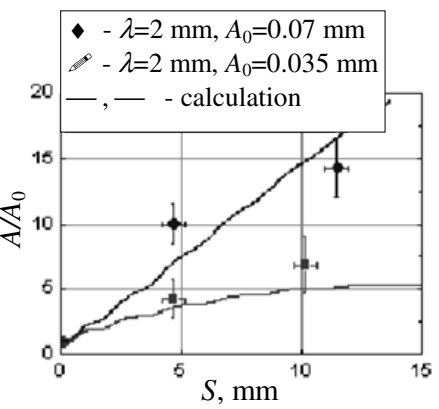

Heterogeneous model

Fig. 5. Perturbation growth in copper sample. Experiment and calculation.

different temperatures and, respectively, different shear strengths. Section of this medium is schematically depicted in Fig. 4.

It is assumed that the period of overheated layers $L$ is kept to be constant, and the width of layer $l$ can be determined by the equation

$$
\begin{aligned}
l & =-\frac{L}{2}\left(\frac{1-\frac{L}{l}}{\tau}+\frac{\dot{T}}{\beta\left(T_{m}-T\right)}\right) \\
\tau & =\frac{L^{2}}{\chi}
\end{aligned}
$$

$T_{m}$ is the melting temperature; $\chi$ is the coefficient of temperature conductivity.

The total system of equations of the two-temperature model of heterogeneous deformation has the form:

$$
\begin{array}{cl}
\dot{Y}_{d}=3 G \dot{\varepsilon}-\left(1-\frac{\omega}{\delta}\right) \cdot \frac{Y_{d}-Y_{S}}{\tau}-\frac{\omega}{\delta} \cdot \frac{\tilde{Y}_{S}}{\tilde{\tau}} \cdot \frac{G}{\tilde{G}} & \tilde{Y}_{S}=Y_{S}(T+\Delta T) \\
\Delta \dot{T}=(\delta-1) \cdot \dot{T}-\frac{\delta^{3}}{(\delta-1) \cdot \tau_{T}} \cdot \Delta T & \tilde{G}=G(T+\Delta T)) \\
\dot{\delta}=\frac{\delta^{2}}{2}\left(\frac{T \cdot}{\beta\left(T_{m}-T\right)}+\frac{1-\delta}{\tau_{T}}\right) & \delta(t)=\frac{L}{l} \\
\end{array}
$$

All parameters with "wave" correspond to overheated layers. Coefficient $\omega$ determines volume fraction of substance, which contains heterogeneous structures. $\omega$ and $L$ depend on strain rate, shock wave 
intensity, initial structure - grain sizes, dislocation density. Unfortunately, in this model, these parameters are not determined by equations, but they are specified as initial parameters. It is a significant disadvantage.

Figure 5 presents results of calculations of pressure and stress intensity behind shock wave front, which were obtained with use of homogeneous and heterogeneous models.

As it is possible to see in the graphs, the heterogeneous model describes actually the experimental results. It should be noted that large number of experiments is required for reliable verification of the model.

\section{Conclusion}

The paper presents models of homogeneous and heterogeneous deformations of metal, as well as methods, which allow obtaining parameters for the mentioned models.

As promising fields for further activities, the following can be outlined:

- direct numerical simulation of high-rate deformation basing on molecular mechanics;

- study of influence of high-rate deformation on properties and structures of metals, including shock-wave hardening and annealing;

- obtainment of data on rheological properties of metals at pressures higher $1 \mathrm{TPa}$.

\section{References}

1. D. Steinberg, S. Cochran, M .Guiuan, A constitutive model for metals applicable at high-strain rait, Joun. of Appl. Phys, vol.51, \# 3, p.1496-1504 (1980)

2. D.J. Steinberg and C.M. Lund, J. Appl. Phys, 651528 (1989)

3. F.J. Zerilli, R.W. Armstong, J. Appl. Phys. Vol.61, \# 5, p.1816-1825 (1987)

4. D.L. Preston, D.L. Tonks and D.C. Wallace, PTW Materials Model, LANL internal memo

5. U.F. Kocks, Constitutive Relations for Slip, In Constitutive Equations in Plasticity, Argon (ed.). 81. MIT Press. Copmbridge, MA (1975)

6. P.S. Follansbee, High-Strain-Rate Deformation of FCC Metals and Alloys, In Metallurgical Application of Shock-Wave and High-Strain-Rate Phenomena, 451, Macel Dekker, NY (1986)

7. D. Steinberg, R. Sharp, Interpretation of shock date for, beryllium and uranium with elasticviscous-plastic constitutive model, Journ. of Appl. Phys, vol.52, \# 8, p.5072-5083 (1981)

8. J. Swegle, D. Gready, Calculation of thermal trapping in shear bands, Metallurgical application of shock wave and high-strain rate phenomena, New-York and Basel, p.705-722 (1986)

9. M.A. Meyers, L.E. Murr, Shock waves and phenomena of high-rate deformation of metals, M.: Metallurgiya, 512 p. (1984)

10. G.N. Epshtein, Structures of metals deformed by explosion, Moscow, "Metallurgiya" (1980)

11. V.A. Raevsky, A.M. Podurets, V.G. Khanzhin et al, Twin structures in copper after shock and shockless high-rate deformation (Procedings of International Conference "IX Khariton's Scientific Readings", Sarov, RFNC-VNIIEF, p.424, 2007)

12. T. Svenson, Formation of dislocations in pure aluminum under quasi-static and shock loadings. Shock waves and phenomena of high-rate deformation of metals / Edited by M.A. Meyers, L.E. Murr: Transl. from Engl., M.: Metallurgiya, p.164 (1984)

13. S.A. Novikov, O.N. Ignatova, E.V. Koshatova et al, Study of behavior of copper with various grain sizes during dynamic deformation, News of Russian Academy of Missile and Artillery Sciences, \# 4(41) (2004)

14. O.N. Ignatova, A.N. Malyshev, V.A. Raevskiy et al, Peculiarities of high rate deformation of copper upon convergence of cylindrical chanals by action of shock waves (SCCM, 2009), edited by M.L. Elert, W.T. Butter, M.D. Furnish, W.W. Anderson, p.735-738 (2009)

15. V.A. Raevsky, O.N. Ignatova, Energetic model of metal strength (Paper for Conference "New models and hydrocodes...", Paris, May 23-28, 2010) 
16. J.W. Swegle and D.E. Grady, Shock viscosity and the prediction of shock wave rise times, J .Appl. Phys. 58(2), 15 July, p.692-701 (1985)

17. V.A. Ogorodnikov, E.S. Tyun'kin, A.G. Ivanov, Strength and viscosity of metals in wide range of strain rate change, FGV, V.36, \# 3 (1995)

18. L.V. Al'tshuler, S.E. Brusnikin, Equation of state of compressed and heated metals, TVT, \# 1, p.42-51 (1987)

19. E.A. Kozlov, V.I. Tarzhanov, I.V. Telichko, D.M. Gorbachev, D.G. Ponkratov, Relaxation of stresses at elastic precursor in unalloyed uranium and some alloys based on it, Papers of Academy of Sciences, v. 408, \# 3, p.328-332 (2006)

20. O.N. Aprelkov, V.V. Igonin, A.I. Lebedev, V.A. Rayevsky, P.N. Nizovtsev, V.P. Soloviev, Study of Rayleigh-Taylor instability in copper and aluminum at pressures up to 45GPa and at strain rates of $10^{5} \div 10^{8}$ (Procedings of International Conference "V Khariton's Scientific Readings", "Substances, materials and constructions under intensive dynamic loading", Sarov, 2003)

21. A.I. Lebedev, P.N. Nizowtsev, V.A. Rayevsky, V.P. Soloviev, Rayleigh-Taylor instability in Strong Media (Experimental Study, 5-th International workshop on the physics of compressible turbulent mixing, New York, USA, pp.231-236, 18-21 July 1995)

22. P.N. Nizovtsev, V.A. Rayevsky, O.N. Ignatova, Phenomenological model of high-rate heterogeneous deformation of metals. RFNC-VNIIEF Proceedings, issue 7, p.318-329 (2004) 